\title{
Clinical outcome following DIAM implantation for symptomatic lumbar internal disk disruption: a 3-year retrospective analysis
}

\author{
This article was published in the following Dove Press journal: \\ Journal of Pain Research \\ 31 October 2016 \\ Number of times this article has been viewed
}

\author{
Kang Lu' \\ Po-Chou Liliang' \\ Hao-Kuang Wang' \\ Jui-Sheng Chen' \\ Te-Yuan Chen' \\ Ruyi Huang ${ }^{2}$ \\ Han-Jung Chen ${ }^{1,3}$ \\ 'Department of Neurosurgery, E-Da \\ Hospital, ${ }^{2}$ Department of Family \\ Medicine, E-Da Hospital, ${ }^{3}$ Department \\ of Neurosurgery, E-Da Cancer \\ Hospital, College of Medicine, I-Shou \\ University, Kaohsiung, Taiwan
}

Background/objective: Internal disk disruption (IDD), an early event of lumbar disk degeneration, is the most common cause of low back pain. Since increased intradiskal pressure (IDP) is associated with symptoms and progression of disk degeneration, unloading a painful disk with an interspinous process device (IPD) is a rational treatment option. The goal of this study was to evaluate the effectiveness of dynamic stabilization with an IPD in the treatment of symptomatic IDD of the lumbar spine.

Patients and methods: Patients with symptomatic IDD were treated with implantation of an IPD, the device for intervertebral assisted motion (DIAM). Diagnosis of IDD was based on typical MRI finding of posterior annular high-intensity zone and positive provocative test on discography. IDP was analyzed intraoperatively. Axial back and leg pain was evaluated with visual analog scale, functional status with Oswestry Disability Index, and final clinical outcomes with Odom criteria. Data from 34 patients followed up for at least 3 years were collected.

Results: DIAM implantation significantly reduced IDP $(\mathrm{n}=11, P<0.0001)$. All 34 patients reported symptom relief. Thirty-one patients $(91 \%)$ remained symptom free until the last followups. Three patients ( $9 \%$ ) experienced recurrence of pain, of which the causes were unrelated to the IDD or surgery. Disk status at the DIAM-implanted segments remained stable. Segmental flexion/extension mobility was preserved in 27 of 30 patients with preoperative mobility. No proximal or distal adjacent segment degeneration was observed. The final clinical outcomes were excellent/good in 31 and fair/poor in three patients.

Conclusion: For patients with symptomatic IDD, dynamic stabilization with DIAM provides pain relief and functional improvement. The implantation maintains disk status and prevents progression of disk degeneration, without compromising segmental flexion/extension mobility or causing adjacent segment degeneration.

Keywords: internal disk disruption, lumbar disk degeneration, DIAM, interspinous process device, intradiskal pressure

\section{Introduction}

Internal disk disruption (IDD) has been found to be the most prevalent cause of chronic low back pain (LBP), surpassing facet joint and sacroiliac joint pain. ${ }^{1,2}$ Even in the absence of nerve root involvement, IDD can cause pain in the back and leg. ${ }^{3}$ Though somatic LBP is the major presentation, referred thigh pain or leg pain, when present, may be misleading in diagnosis and treatment. ${ }^{4,5}$
Department of Neurosurgery, E-Da

Hospital, College of Medicine, I-Shou

University, I Yida Road, Yanchau District,

Kaohsiung 824, Taiwan

Tel +88676I5 00II Ext 2976

Email kanglu.1002@gmail.com 
IDD is regarded as an early event of disk degeneration. ${ }^{6}$ The associated pain is believed to be related to ingrowth of nerves and blood vessels through an annular fissure. ${ }^{7-10}$ Such LBP tends to be chronic and persistent over time. ${ }^{6}$ Despite the fact that IDD has been identified as a common cause of disabling LBP in young patients, ${ }^{9}$ there is little consensus as to what the best treatment is. ${ }^{8}$

Because mechanical strains and elevated intradiskal pressure (IDP) have been shown to be closely associated with the progression of lumbar disk degeneration, ${ }^{10-12}$ unloading a painful disk in its early stage of degeneration would theoretically alleviate pain and even stop or slow down the degenerative process. A recent development in the surgical treatment of lumbar degenerative diseases is the application of interspinous process devices (IPDs). ${ }^{13-15}$ However, little is known about the feasibility and efficacy of IPDs in the treatment of symptomatic IDD.

The aim of this retrospective study was to evaluate the efficacy of implanting an IPD, the device for intervertebral assisted motion (DIAM, Medtronic Inc., Memphis, TN, USA) implant, as the surgical treatment for patients suffering from refractory symptomatic IDD, with at least 3 years of follow-up.

\section{Patients and methods}

\section{Patient selection}

Between November 2008 and December 2012, 52 patients with symptomatic IDD were selected as candidates for DIAM implantation. Before considering surgery, all patients had received at least 3 months (mean 9 months, range 3-60 months) of failed nonsurgical treatments, which included lifestyle and work load modifications, bracing, and continuous physical therapy. Twenty-six patients had also received one or more sessions of injection or radiofrequency therapy. The treated sites were sacroiliac joints in 17 , disks in eight, facet joints in five, and unknown in three patients. All patients felt that their quality of life was significantly affected by the symptoms, and they actively sought for more aggressive measures. None of them had history of malignancy, psychiatric disorders, or previous spinal trauma, infection, or surgery.

The major symptom was axial low back/buttocks pain with or without leg pain. The axial pain/leg pain ratios according to patients' own perceptions were $100 / 0$ in six, $90 / 10$ in seven, 80/20 in six, 70/30 in eight, 60/40 in three, and 50/50 in four cases. Corresponding neurologic deficits were detected in 13 cases, manifested as motor weakness with or without sensory impairment. None of the patients had intermittent claudication typical of lumbar spinal stenosis or symptoms and physical findings suggestive of hip joint or sacroiliac joint arthropathy.

The diagnosis of IDD was based on imaging studies and provocative discography. Excluding five patients who refused discography and nine who showed negative discographic findings or reported no concordant pain on provocative tests, a total of 34 patients (14 men and 20 women) with a mean age of 38 years (range 24-51) were included in this retrospective study (Table 1). The study protocols were approved by the Institutional Review Board of the E-Da Hospital.

\section{Image criteria}

All patients received anteroposterior, lateral flexion/extension radiographs and MRI. Images were analyzed by the operating surgeon and by an independent neuroradiology specialist. MR findings of dark signal with a high-intensity zone in the posterior annulus were regarded as diagnostic of IDD. ${ }^{16}$ Meanwhile, the Pfirrmann classification was used for assessing the severity of disk degeneration. ${ }^{17}$ According to the Pfirrmann criteria, five cases were classified as grade II and 29 as grade III.

Excluded were patients who showed image findings of disk extrusion or sequestered herniation, advanced disk

Table I Patient demographics and preoperative MRI findings $(n=34)$

\begin{tabular}{ll}
\hline Demographic data & Values \\
\hline Age (years) & 38 (range 24-5I) \\
Male/female & $14 / 20$ \\
Levels operated & \\
L3-4 & 2 \\
L4-5 & 25 \\
L5-6 & 2 \\
L3-4, L4-5 & 4 \\
L4-5, L5-6 & 1 \\
Symptomatology & \\
Back pain only or predominant & \\
Back pain/leg pain equivalent & 27 \\
Leg pain only or predominant & 7 \\
VAS & 0 \\
ODI & 7.6 (range 5-10) \\
MRI findings of treated disks & 25.2 (range 18-36) \\
Inhomogenous gray signal & \\
Slightly decreased disk height & \\
Annular fissure & \\
Foraminal compromise &
\end{tabular}

Notes: aPresence of a sixth lumbar vertebra with intact lamina and spinous process; 'Back pain or leg pain predominance was based on every patient's own perception of the locations of pain; 'Back pain/leg pain ratios were $100 / 0$ in 6, 90/10 in 7, 80/20 in 6, $70 / 30$ in 8 patients; 'Leg pain as the only or major symptom; ${ }^{\circ}$ Compatible with grade III disk degeneration based on Pfirrmann's MR classification ( I7); ${ }^{f}$ Hyperintense signal within the posterior annulus fibrosus, shown on T2-weighted MR images; ${ }^{8}$ Associated with annular bulging without protrusion or extrusion of nucleus pulposus.

Abbreviations: VAS, visual analog scale; ODI, Oswestry Disability Index; MR, magnetic resonance; MRI, magnetic resonance imaging. 
degeneration with Modic changes, disk space collapse (Pfirrmann grades IV or V), defects of pars interarticularis, spondylolisthesis, lumbar stenosis, or disease at the L5-S1 level. Direct decompression with or without fusion was our option of choice for such patients.

\section{Surgical procedure}

All operations were performed by the same surgeon (KL). After midline skin incision, the paraspinal muscles were dissected and retracted to expose the target rostral and caudal spinous processes and laminae. The interspinous and the interlaminar ligaments were removed, while the supraspinous ligament was preserved. Epidural fat, if present, was kept as intact as possible. The DIAM implant was placed between the two spinous processes and laminae under the supraspinous ligament. The implant size was determined by insertion trials ( $8 \mathrm{~mm}, 10 \mathrm{~mm}, 12 \mathrm{~mm}$, and $14 \mathrm{~mm}$ ). The implant was firmly anchored in place by the two tethers passed around the two adjacent spinous processes, and fixed with crimps.

\section{IDP measurement}

IDP was measured intraoperatively in the first 14 cases using an intracranial pressure monitoring device (Codman Microsensor ICP Transducer; Codman \& Shurtleff, Inc., Raynham, MA, USA). Written informed consent was obtained from patients to participate in the study. After removing the interlaminar ligament, a limited laminotomy was performed under an operating microscope to expose the nerve root. The nerve root was gently retracted medially. A small puncture of the annulus fibrosus was made with an $18 \mathrm{G}$ needle or a No. 11 scalpel blade. The tip of the microsensor was inserted through the annulus into the center of the disk. A baseline resting IDP reading was obtained first, and then subsequently at three different time points, including distracting between the spinous processes and laminae with a distractor, inserting trial templates, and after DIAM implantation (Figure 1). The sensor was removed before wound closure.

\section{Follow-up assessments}

All patients were followed up at 3 weeks, 3 months, 6 months, 12 months, and then annually after surgery. Back and leg pain intensity was assessed with the visual analog scale (VAS) and severity of functional impairment with the Oswestry Disability Index (ODI). Final clinical outcomes were evaluated with the Odom criteria.

Radiographs were obtained at each follow-up to evaluate the implant position, status of the disk, segmental mobility at the DIAM-implanted level, and the proximal and distal adjacent disk levels.

\section{Statistical analysis}

Preoperative and postoperative data of VAS and ODI were compared using the paired $t$-test. Comparisons of IDP data among intraoperative events were made using the ShapiroWilk test. All analyses were performed with SPSS 15 (SPSS Inc., Chicago, IL, USA).
A

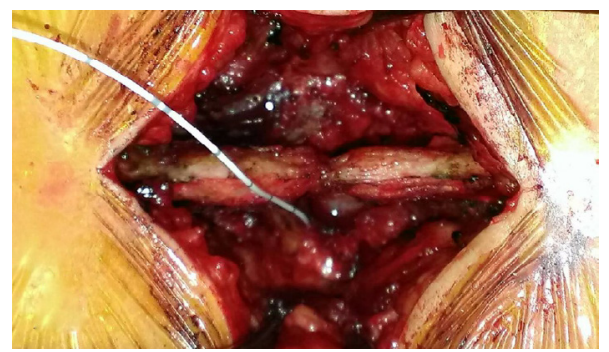

C

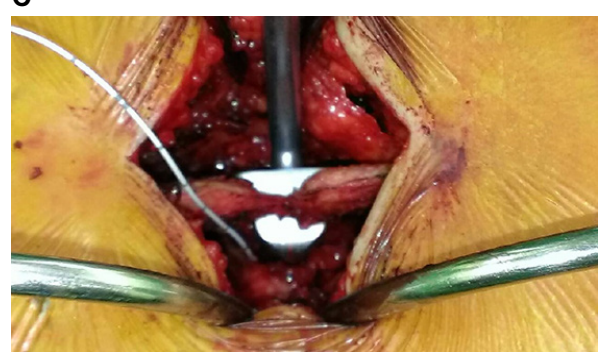

B

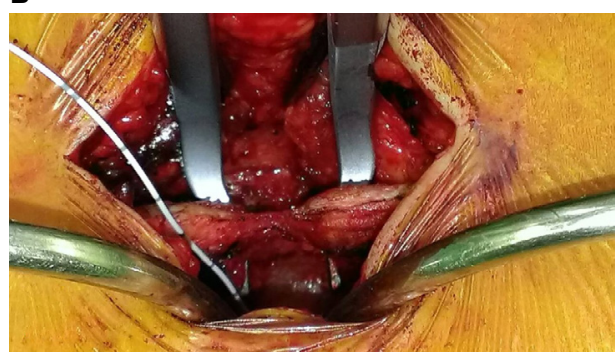

D

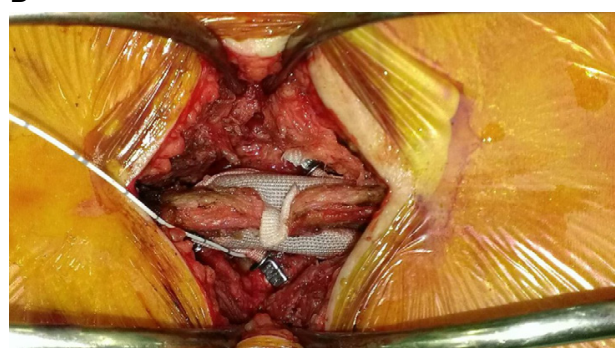

Figure I Intraoperative IDP recording using a microsensor for intracranial pressure monitoring.

Note: The monitoring was done at a resting state (A), on distraction between the two spinous processes with a distractor (B), a template (C), and after DIAM implantation (D). Abbreviations: DIAM, device for intervertebral assisted motion; IDP, intradiskal pressure. 


\section{Results IDP data}

IDP monitoring was successfully done in eleven patients and abandoned in three patients. The reasons for aborting pressure monitoring included failure to pass the sensor through the annulus and mechanical problems of the sensors or monitors. The mean resting pressure before any interspinous distraction was $267.2 \pm 37.7 \mathrm{mmHg}$. Distraction between spinous processes with the distractor caused an abrupt drop of pressure (mean $81.5 \pm 34.0 \mathrm{mmHg}$ ). The pressure rebounded on replacing the distractor with a template (mean $0.6 \pm 57.7 \mathrm{mmHg}$ ). The mean final pressure after securing the DIAM implant was 91.9 $\pm 38.7 \mathrm{mmHg}$. Compared to the initial resting IDP, the readings recorded on distraction, template insertion, and DIAM implantation were all significantly lower $(P<0.0001$; Figure 2$)$.

As the pattern of intraoperative IDP changes was so distinctive and constant, pressure monitoring was not performed in the rest of the cases.

\section{Illustrative case}

A 28-year-old woman presented with LBP with radiation to buttocks for 3 years. The pain was dull and persistent. Soreness was felt on her buttocks and posterior thighs. The pain did not radiate to lower legs. There was no numbness on the legs. Her tolerance to upright positions, including standing and sitting, was poor. Her ability to walk was normal. Her perception of pain distribution was $80 \%$ on the low back and buttocks, and $20 \%$ on the legs. Her VAS was 6-8, depending on the time of day. Her ODI was 36 . She showed normal posture and gait pattern. Neurological examination disclosed

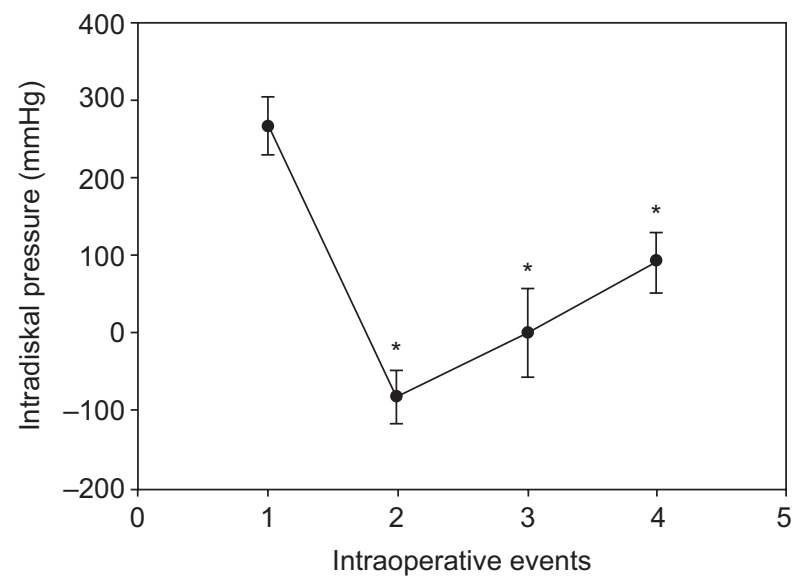

Figure 2 Mean IDP readings at different stages during surgery.

Notes: I, baseline; 2, spinous processes under distraction with a distractor; 3, a template inserted; 4, DIAM implanted between spinous processes. Data are presented as mean $\pm S D(n=9)$. $* P<0.0001$.

Abbreviations: DIAM, device for intervertebral assisted motion; IDP, intradiskal pressure; SD, standard deviation. no motor or sensory deficits. She had received radiofrequency therapy twice at two other hospitals, 1 year and 2 months prior to her visit, respectively. Both sessions gave her temporary pain relief, but symptoms soon recurred.

Radiographs showed that she had a sixth lumbar vertebra, and that there was a loss of L5-6 disk height (Figure 3A-C). MRI showed dark signal in the disk with a posterior highintensity zone (Figure 3D and E). As the L6 level possessed lamina and spinous process that were large enough, a DIAM implant was secured between L5 and L6. She reported relief of the LBP and leg pain immediately after surgery. She remained symptom-free at the 3-year follow-up. Postoperative dynamic radiographs showed preserved flexion/extension mobility and enlarged neuroforamen (Figure 3F-H).

\section{Clinical outcome}

All 34 patients reported improvement of pain and functional status at the first follow-up 1 week after surgery. Thirty-one cases $(91 \%)$ remained free of symptoms and of any requirement for medications until the last follow-up. Mean preoperative VAS and ODI were 7.8 \pm 1.5 and $24.6 \pm 3.5$, while values at the last follow-ups were $1.3 \pm 2.2$ and $6.3 \pm 7.6$, respectively $(P<0.0001)$ (Figure 4).

Three patients reported recurrence of back and/or leg pain during follow-ups despite an initial improvement after surgery. MRI was repeated in these patients, which unequivocally revealed resolution of the posterior annular high-intensity zone and widened neuroforamens (Figure 5). The back pain in the first case, a 45-year-old man who underwent L3-4, L4-5 DIAM implantations, started after a fall in a motor vehicle accident. It soon subsided with medical treatment. The second case, a 40-year-old man, suffered new right leg pain caused by L5-S1 disk herniation after relief of LBP following L4-5 DIAM implantation. Microdiskectomy had to be performed 4 months after the initial operation to relieve his symptoms. The third case, a 42-year-old woman, despite initial pain relief after surgery, suffered from recurrence of back and leg pain 3 months later. Imaging and electrophysiological studies did not show any abnormalities. Medications and physical therapy failed to relieve the pain. Meanwhile, she had sleep disorder and menstrual irregularity and visited other specialists for narcotics frequently. The cause of her pain was suspected as psychiatric. A consultation with a psychiatrist was proposed, but refused by the patient. This was the only case who reported poor clinical outcome.

Overall, based on the Odom's criteria, the final clinical outcomes were excellent in 24 cases, good in seven, fair in two, and poor in one case. 

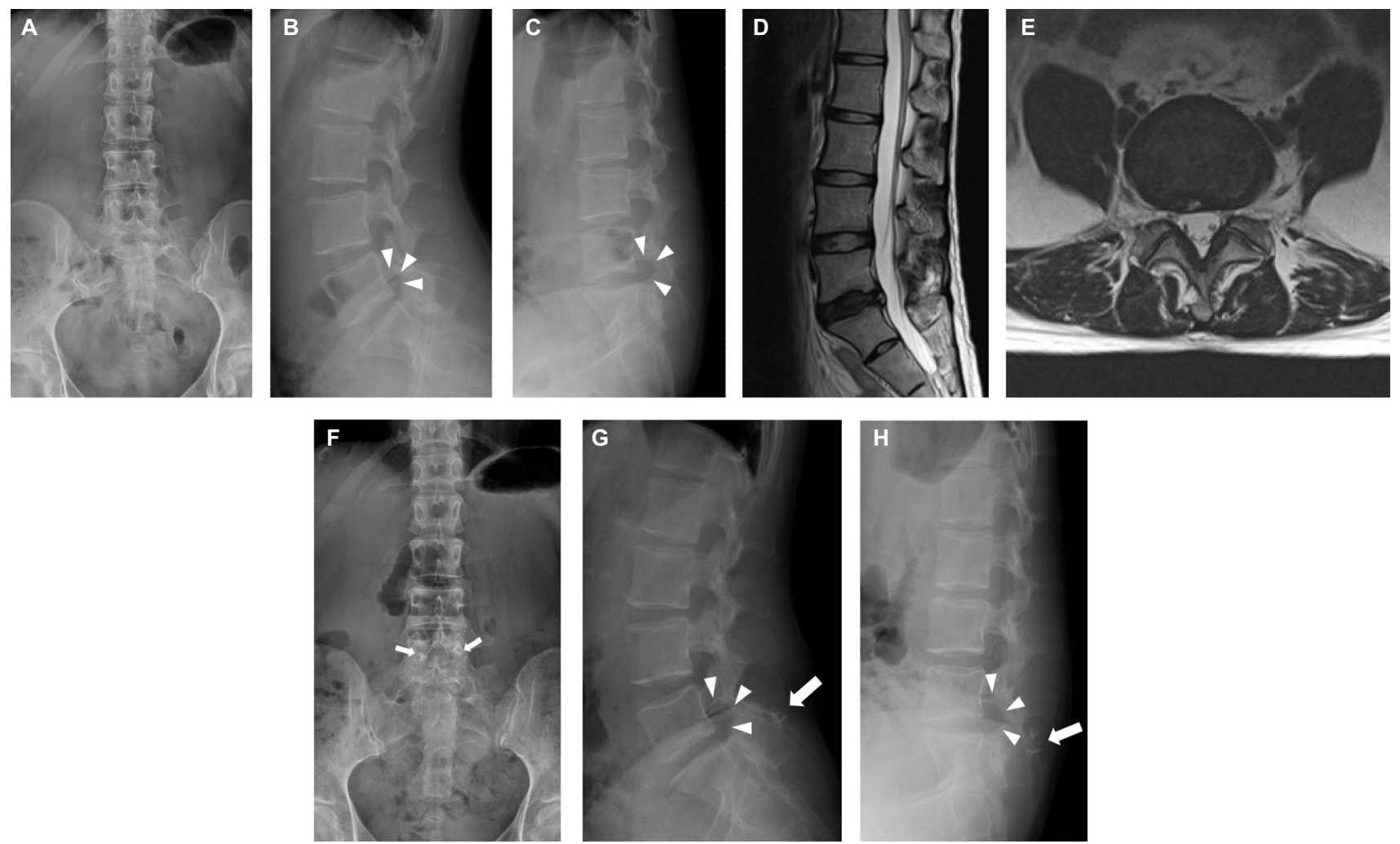

Figure 3 Images of a 28-year-old woman with symptomatic IDD.

Notes: Preoperative radiographs showed presence of a sixth lumbar vertebral and loss of L5-6 disk height (A-C). MRI showed a dark disk with a posterior high-intensity zone (D, E). After DIAM implantation, postoperative radiographs showed preserved segmental flexion/extension mobility and increased neuroforaminal size (arrowheads) $(\mathbf{G}, \mathbf{H})$. The presence of the DIAM implant is indicated by the crimps (arrows in $\mathbf{F}-\mathbf{H}$ ) that fix the tethers of the implant.

Abbreviations: DIAM, device for intervertebral assisted motion; IDD, internal disk disruption; MRI, magnetic resonance imaging.

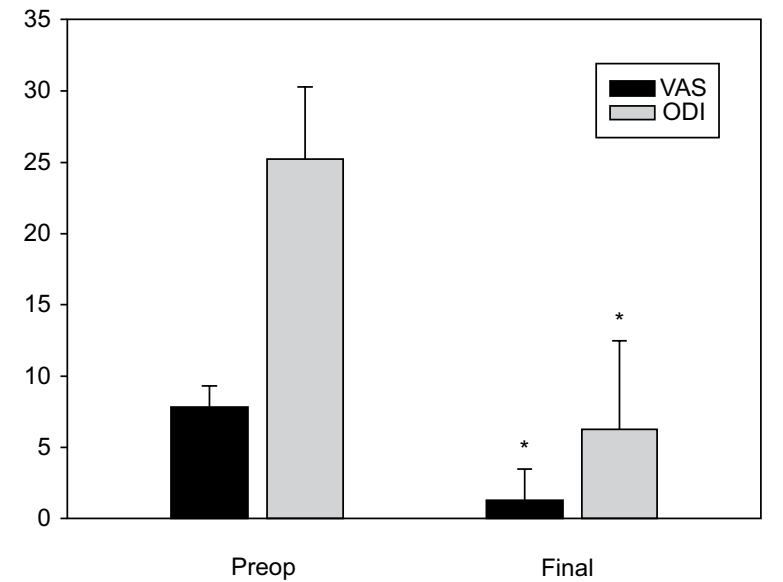

Figure 4 Preoperative and final mean VAS and ODI levels.

Note: Data are presented as mean $\pm S D$. $* P<0.0001$.

Abbreviations: VAS, visual analog scale; ODI, Oswestry Disability Index; SD, standard deviation.

\section{Radiographic outcome}

At the DIAM-implanted segments, follow-up radiographs showed no progression of disk height loss, endplate changes, appearance of vacuum clefts, or segmental instability. Before surgery, obvious segmental flexion/extension mobility was present in 30 cases. At the final follow-up, this was preserved in 27 cases, but restricted in two, and lost in one case. The four cases without obvious preoperative flexion/extension mobility remained the same. There was no radiographic evidence of degeneration at any proximal or distal adjacent segments next to the DIAM-implanted segments.

Repeated MRI was obtained in the three aforementioned cases experiencing recurrent pain. Consistently, all the MR images showed increased neuroforaminal widths and resolution of annular fissures at the DIAM-implanted segments (Figure 5).

\section{Complications}

No fracture of spinous processes or dural tear occurred during the surgical procedures. No patient developed neurologic deficit after surgery. Superficial wound infection occurred in one patient, which subsided with proper wound care. All patients were ambulatory in a lumbar corset on the day of surgery or the next day. Hospital stay was no more than 3 days in all cases.

\section{Discussion}

Different from more advanced lumbar disk degeneration, IDD lacks obvious image findings to justify any aggressive treatment. Even if a patient suffers from severe symptoms, many 

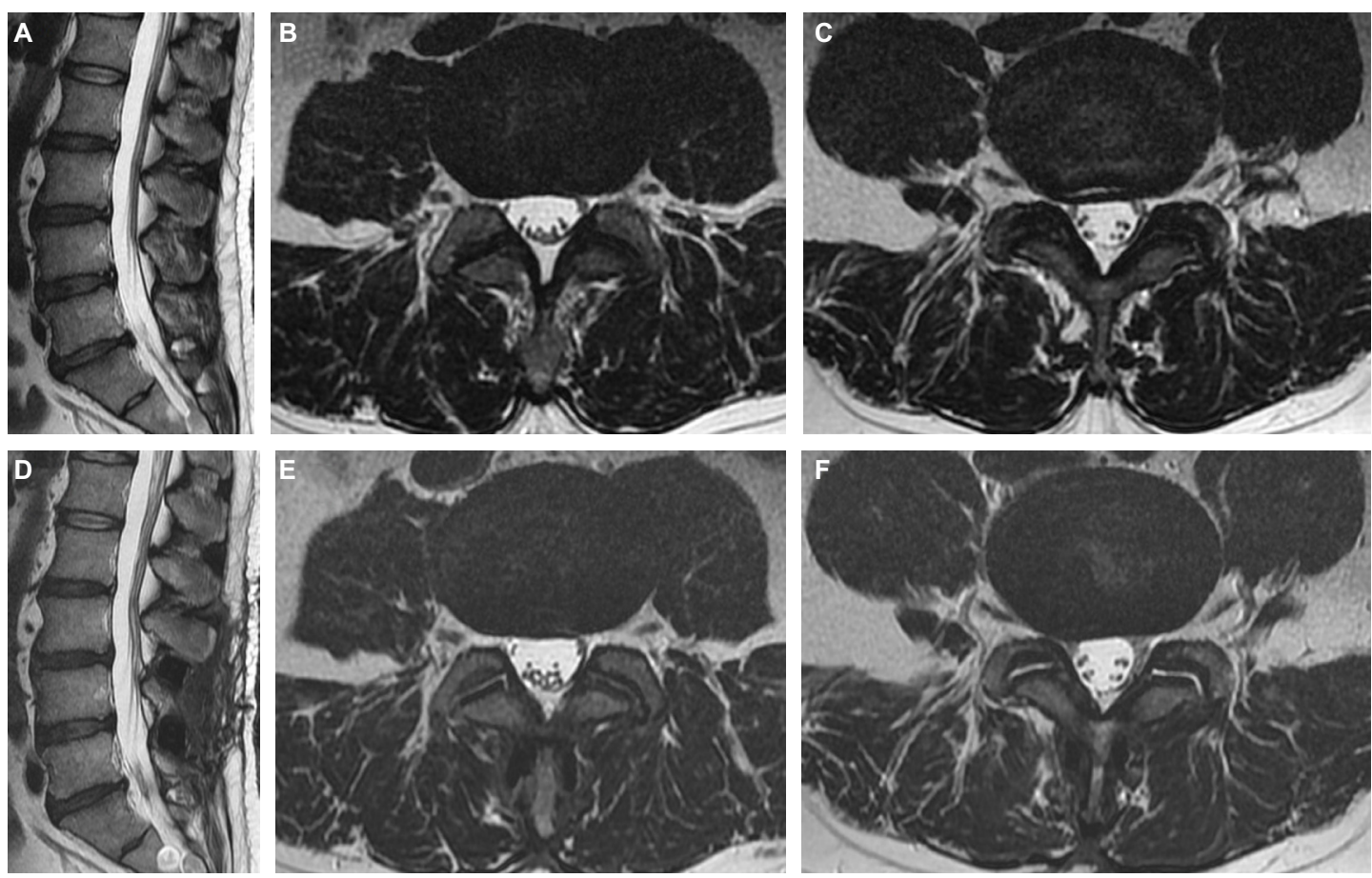

Figure 5 MRI images before and after DIAM implantation.

Notes: Preoperative (A-C) and postoperative (D-F) MRI in a 45-year-old man who underwent DIAM implantation at the L3-4, L4-5 levels. Axial sections through the L3-4 disk (B, E) showed widened bilateral neuroforamens for exiting nerve roots. Sections through the L4-5 disk (C, F) showed resolution of the posterior annular highintensity zone.

Abbreviations: DIAM, device for intervertebral assisted motion; MRI, magnetic resonance imaging.

would not view them as candidates for surgery. However, IDD may cause chronic disabling symptoms, and may progress to more advanced degeneration. ${ }^{6}$ The idea of easing a patient's symptoms, making their quality of life better, and halting the process of degeneration in its early stage is appealing.

We selected only those who presented with predominantly axial low back/buttock pain with no or relatively mild leg symptoms as candidates for stand-alone DIAM implantation. The diagnosis of IDD was based on MRI and discographic findings. Our results indicate that DIAM implantation is an effective and durable surgical option for IDD. We excluded patients with advanced disk degeneration (Pfirrmann grades IV and V), overt lumbar stenosis, spondylolisthesis, and instability. The rationale was the belief that the DIAM implant provides axial distraction but does not relieve anteroposterior or circumferential compression from hypertrophic ligamentum flavum and facet joints present in advanced disk degeneration and lumbar stenosis. Moreover, such cases often have associated disk space collapse and ankylosis of the facet joints, which would significantly limit the applicability of the DIAM implant and its mobility after implantation.

It is arguable whether an intervertebral disk in its early stage of degeneration should not be treated surgically at all. One of the major findings of this study is that in IDD, the symptoms can be severe enough to affect quality of life badly, even though the disk may appear only mildly degenerated. ${ }^{9}$ While waiting for a longer period for conservative treatment to work is always an option, the patient may have to continue tolerating the symptoms and futile therapy. In addition, there is the risk of progressive degeneration and dysfunction of the disk and facet joints over time to a state where dynamic stabilization with a DIAM implant would no longer be optimal, and fusion might become the only choice.

The patterns of IDP changes were strikingly similar among the measured cases. The data provide biomechanical evidence supporting our treatment. Distraction between two adjacent spinous processes and laminae, whether from a distractor, a trial template, or a DIAM implant, unequivocally reduced IDP (Figure 2). Cadaveric ${ }^{18}$ and finite element studies ${ }^{19}$ have demonstrated that IPDs significantly unload implanted intervertebral disks. Given the association between elevated IDP and the progression of disk degeneration, ${ }^{11,12}$ our findings imply disk unloading as a mechanism for the clinical benefits of DIAM implantation.

The maintenance of disk height on follow-up radiographs suggests that the DIAM implant was effective in preventing the progression of disk degeneration. Besides, its limitation on flexion/extension mobility appears minimal, provided such 
mobility is present before surgery. Although one biomechanical feature of IPDs is to act as an "extension block" ${ }^{20}$ it was not obvious in our cases. This might have been due to the relatively young age of our patients, as well as the elastic and compressible property of the silicon-based material of the DIAM implant.

The absence of any adjacent segment degeneration, either proximal or distal, suggests that DIAM implantation has little adverse impact on adjacent segments. This is in accordance with previous reports showing that IPDs do not alter the loading at the adjacent segments. ${ }^{15,18,19,21-25}$ Even in the four patients with no preoperative segmental flexion/extension mobility, and the three cases who lost normal mobility after surgery, radiographic changes of adjacent segment degeneration were not observed. It is likely that even if a segment is immobilized by a DIAM implant, it does not become "fixed". Therefore, the impact on its adjacent segments should be quite different from that caused by rigid fusion.

According to a recent review, placement of IPDs is associated with relatively high complication and failure rates. ${ }^{26}$ Common complications included spinous process fractures and dural tears. Reasons for revision surgery were lack of improvement, worsening of pain, or implant dislocatioin. ${ }^{26}$ In contrast, none of these adverse events occurred in our patients. Several factors might explain the difference. First, our patients were relatively young with very low risk of osteoporosis. Second, an operating microscope was routinely used for removal of the interspinous and interlaminar ligaments, while preserving the epidural adipose tissue if present. This practice should have helped preventing violation of the dura.

Our study has several limitations. First, the number of cases was relatively small. However, the small case number actually reflects our stringency in patient selection. We virtually treated only cases showing early disk degeneration but suffering intractable symptoms. Second, even though MRI and discographic evidence was available for diagnosis, other sources of pain, such as the facet joints and the sacroiliac joints, might have probably existed in some patients. We did not take specific diagnostic measures to exclude them as pain sources. Third, we are unable to provide data concerning facet status before and after surgery. In this regard, even though we have provided evidence of disk unloading (IDP reduction) as underlying the DIAM-mediated symptom relief, we cannot be sure whether facet joint unloading also played a role, though it has been demonstrated in cadavers that IPDs reduce facet loading. ${ }^{25}$ Finally, postoperative MRI was done in only the three patients who had recurrence of pain. The National Health Insurance Administration of our country dictates that MRI done without a just cause is liable to be denied reimbursement or even fined. As the causes of recurrent pain were proved to be unrelated to the initial disk degeneration, the MR findings of resolution of annular fissure and enlargement of neuroforamens can be regarded as image evidence supporting the benefits of DIAM implantation for IDD, even though the number of cases was small.

\section{Conclusion}

In IDD with intractable symptoms, DIAM implantation provides pain relief, functional improvement, and satisfactory clinical outcomes. Such dynamic stabilization appears effective in resolving annular fissures and preventing progression of disk degeneration. Segmental flexion/extension mobility, when present, tends to be maintained. The implant does not lead to either proximal or distal adjacent segment degeneration. These beneficial effects, together with disk unloading and increased neuroforaminal dimensions, may be the mechanisms for the observed satisfactory clinical outcome.

\section{Disclosure}

The authors declare no conflicts of interest in this work.

\section{References}

1. DePalma MJ, Ketchum JM, Saulo T. What is the source of chronic low back pain and does age play a role? Pain Med. 2011;12(2): 224-233.

2. Izzo R, Popolizio T, D’Aprile P, Muto M. Spinal pain. Eur J Radiol. 2015;84(5):746-756.

3. Crock HV. A reappraisal of intervertebral disc lesions. Med J Aust. 1970;1(20):983-989.

4. Laplante BL, Ketchum JM, Saullo TR, DePalma MJ. Multivariable analysis of the relationship between pain referral patterns and the source of chronic low back pain. Pain Physician. 2012;15(2):171-178.

5. Schwarzer AC, Aprill CN, Derby R, Fortin J, Kine G, Bogduk N. The prevalence and clinical features of internal disc disruption in patients with chronic low back pain. Spine. 1995;20(17):1878-1883.

6. Peng B, Fu X, Pang X, et al. Prospective clinical study on natural history of discogenic low back pain at 4 years of follow-up. Pain Physician. 2012;15(6):525-532.

7. Ito K, Creemers L. Mechanisms of intervertebral disk degeneration/ injury and pain: a review. Global Spine J. 2013;3(3):145-152.

8. Peng BG. Pathophysiology, diagnosis, and treatment of discogenic low back pain. World J Orthop. 2013;4(2):42-52.

9. Stefanakis M, Al-Abbasi M, Harding I, et al. Annulus fissures are mechanically and chemically conductive to the ingrowth of nerves and blood vessels. Spine. 2012;37(22):1883-1891.

10. Sehgal N, Dortin JD. Internal disc disruption and low back pain. Pain Physician. 2000;3(2):143-157.

11. Adams MA, McNally DS, Dolan P. "Stress" distribution inside intervertebral discs. The effects of age and degeneration. J Bone Joint Surg Br. 1996;78(6):965-972.

12. Tsantrizos A, Ito K, Aebi M, Steffen T. Internal strains in healthy and degenerated lumbar discs. Spine. 2005;30(19):2129-2137. 
13. Fabrizi AP, Maina R, Schiabello L. Interspinous spacers in the treatment of degenerative lumbar spinal disease: our experience with DIAM and Aperius devices. Eur Spine J. 2011;20(suppl 1): S20-S26.

14. Sandu N, Schaller B, Arasho B, Orabi M. Wallis interspinous implantation to treat degenerative spinal disease: description of the method and case series. Expert Rev Neurother. 2011;11(6):799-807.

15. Sur YJ, Kong CG, Park JB. Survivorship analysis of 150 consecutive patients with DIAM implantation for surgery of lumbar spinal stenosis and disc herniation. Eur Spine J. 2011;20(2):280-288.

16. Finch P. Technology insight: imaging of low back pain. Nat Clin Pract Rheumatol. 2006;2(10):554-561.

17. Pfirrmann CWA, Metzdorf A, Zanetti M, Hodler J, Boos N. Magnetic resonance classification of lumbar intervertebral disc degeneration. Spine. 2001;26(17):1873-1878.

18. Swanson KE, Lindsey DP, Hsu KY, Zucherman JF, Yerby SA. The effects of an interspinous implant on intervertebral disc pressure. Spine. 2003;28(1):26-32.

19. Bellini CM, Galbusera F, Raimondi MT, Mineo GV, Brayda-Bruno M. Biomechanics of the lumbar spine after dynamic stabilization. J Spinal Disord Tech. 2007;20(6):423-429.
20. Park SW, Lim TJ, Park J. A biomechanical study of the instrumented and adjacent lumbar levels after In-Space interspinous spacer insertion. J Neurosurg Spine. 2010;12(5):560-569.

21. Caserta S, La Maida GA, Misaggi B, et al. Elastic stabilization alone or combined with rigid fusion in spinal surgery: a biomechanical study and clinical experience based on 82 cases. Eur Spine J. 2002;11(suppl 2): S192-S197.

22. Lu K, Liliang PC, Wang HK, et al. Reduction in adjacent-segment degeneration after multilevel posterior lumbar interbody fusion with proximal DIAM implantation. J Neurosurg Spine. 2015;23(2):190-196.

23. Senegas J. Mechanical supplementation by non-rigid fixation in degenerative intervertebral lumbar segments: the Wallis system. Eur Spine J. 2002;11(suppl 2):S164-S169.

24. Whitesides TE Jr. The effect of an interspinous implant on interverbral disc pressures. Spine. 2003;28(16):1906-1907.

25. Wiseman CM, Lindsey DP, Fredrick AD, Yerby SA. The effect of an interspinous process implant on facet loading during extension. Spine. 2005;30(8):903-907.

26. Gazzeri R, Galarza M, Neroni M, et al. Failure rates and complications of interspinous process decompression devices: a European multicenter study. Neurosurg Focus. 2015;39(4):E14.
Journal of Pain Research

\section{Publish your work in this journal}

The Journal of Pain Research is an international, peer reviewed, open access, online journal that welcomes laboratory and clinical findings in the fields of pain research and the prevention and management of pain. Original research, reviews, symposium reports, hypothesis formation and commentaries are all considered for publication.

\section{Dovepress}

The manuscript management system is completely online and includes a very quick and fair peer-review system, which is all easy to use. Visit http://www.dovepress.com/testimonials.php to read real quotes from published authors. 